\title{
Omission training (negative automaintenance) in the rat: Effects of trial offset
}

\author{
C. M. LOCURTO \\ College of the Holy Cross, Worcester, Massachusetts 01610 \\ H. S. TERRACE \\ Columbia University, New York, New York 10027 \\ and \\ JOHN GIBBON \\ Columbia University and The New York State Psychiatric Institute, New York, New York 10027
}

\begin{abstract}
The role of trial offset in omission training was studied in hooded rats using two types of omission training procedures. In both procedures a lever contact during a trial canceled food delivery at the end of the trial. In one omission procedure trials were of fixed duration $(15 \mathrm{sec})$, whereas in the second omission procedure a lever contact terminated the trial (retracted the lever). Each omission subject was yoked with a second subject. Additional groups of rats were exposed to one of two types of random control procedures. In one random control procedure a contact with the lever terminated the trial, and in a second procedure trial duration was fixed. Higher response probabilities were generated by the omission procedure in which trials were terminated by lever contacts than by the fixed-trial omission procedure. No differences were observed between the two random control procedures.
\end{abstract}

A number of studies have focused upon the extent to which autoshaping may represent an exception to the law of effect (e.g., Hearst \& Jenkins, 1974). Perhaps the soundest empirical basis for this assertion is derived from studies of omission training in which responses prevent delivery of the reinforcer. As investigated initially by Williams and Williams (1969), food was presented to pigeons at the end of every trial except those trials during which a keypeck occurred. The first response not only canceled food but also terminated the trial.

The presumed absence of a positive responsereinforcer (R-S*) relation during omission training (also referred to as "negative automaintenance") is the basis for the suggestion that the procedure serve as an assay of the control exerted by stimulus-reinforcer (S-S*) relations over skeletal responses (Gamzu \& Schwam, 1974; Stiers \& Silberberg, 1974; cf. Locurto, Terrace, \& Gibbon, 1976). It has been argued, however, that omission training may contain sources of reinforcement for responding by providing the necessary conditions for the development of trial offset as a conditioned reinforcer. On trials during which no responses occur, trial offset is followed by food (e.g., Herrnstein \&

This research was performed while the first author was a postdoctoral research associate in the Stimulus Control Laboratory, Columbia University (H. S. Terrace, Director). It was, in part, supported by grants from the National Science Foundation (GB 03781 and GB 34095) and the National Institute of Health (HD 00930). Portions of this paper were presented at the meeting of the Eastern Psychological Association, New York, New York, 1975
Loveland, 1972; Hursh, Navarick, \& Fantino, 1974; cf. Peterson, Lyon, Stone, \& Scott, 1977).

Previous studies using pigeons have yielded conflicting results with respect to the reinforcing properties of trial offset during omission training (compare, for example, Brownstein \& Balsam, 1975; Peterson et al., 1977; Schwartz, 1972; with Herrnstein \& Loveland, 1972; Hursh et al., 1974). The present study evaluated the reinforcing properties of trial offset under a broader set of conditions than have been used in previous studies of omission training. Using rats, two types of omission procedures were compared: one in which trials were terminated by the first lever contact (responseterminated) and a second in which trials were of fixed duration (fixed-trial).

Performance under omission training was assessed via comparison with performance under two types of control procedures. In one control a yoked subject received the same trial durations and the distribution of lever-food pairings as did his omission partner. Two random control procedures were also used (cf. Rescorla, 1967). In both procedures, trials and food were programmed independently of each other. In one random control, a lever contact during a trial terminated the trial; in the second random control, trials were of fixed duration.

\section{METHOD}

\section{Subjects}

The subjects were 32 male hooded rats, approximately 125 days old at the start of training. Throughout the experiment 
they were maintained at $80 \%$ of their free-feeding weights. Supplementary food was provided in the home cages immediately following each session to maintain the $80 \%$ weights.

\section{Apparatus}

Two identical test chambers were used, each a modified ice chest (80-quart capacity). The subject's portion of the chamber was $24 \times 29.5 \times 34 \mathrm{~cm}$ high. The test panel consisted of a feeder dish located in the middle of the panel at floor level. A retractable rodent lever (Camden Instruments, Inc.) housed $4 \mathrm{~cm}$ above the floor and $7 \mathrm{~cm}$ to the right of the dish was the only other piece of equipment on the panel. A force of approximately $15 \mathrm{~g}(.15 \mathrm{~N})$ was necessary to depress the lever sufficiently to register a press. The lever protruded $2.5 \mathrm{~cm}$ into the chamber when inserted.

Contacts with the lever in each chamber were recorded via drinkometer circuits. The reinforcer was a $97-\mathrm{mg}$ standard Noyes rat pellet.

\section{Procedure}

Sixteen subjects were randomly assigned to omission training, while the remaining subjects received random control training. Initially, each subject was trained to eat reliably from the feeder dish. Feeder training consisted of three sessions held on consecutive days. During each session 50 pellets were delivered on a variable-time (VT) 20-sec schedule. All VT schedules were generated using the Fleshler-Hoffman (1962) distribution of intervals.

On the day following the third feeder training session, all subjects were given the first of seven operant-level sessions. Each operant-level session consisted of 25 trials with no reinforcers. A trial was defined as the 15 -sec insertion of the lever into the chamber. ${ }^{1}$ Intertrial intervals (ITIs) were scheduled using a VT 60 -sec tape.

The 35 sessions of training began following the seventh operant-level session. The temporal parameters in effect during omission and random control training were the same as those in effect during operant-level sessions.

Omission training. The 16 omission subjects were divided into eight pairs and members of each pair were randomly placed in the omission or in the yoked condition. Four pairs were then assigned to each of the two omission procedures. In each omission procedure a reinforcer was delivered to both members of a pair at the end of each trial during which the omission subject did not contact the lever. The two omission procedures were(1) Omission/response-terminated trial: The first lever contact during a trial by the omission subject ended the trial (i.e., retracted the lever) and canceled the reinforcer for that trial in both chambers. (2) Omission/fixed trial: Lever contacts by the omission subject had no effect on trial duration, but the first contact by this subject canceled the reinforcer at the end of the trial in both chambers.

Random control training. Sixteen subjects were assigned to either the random control/response-terminated procedure or to the random control/fixed-trial procedure. In each procedure trials and food deliveries were programmed by independent VT schedules.

Sessions were generally conducted 6 days a week.

\section{RESULTS}

The probability of responding for each subject in the response-terminated and the fixed-trial omission groups is shown in Figure 1. The probability of a response was expressed as the proportion of trials with at least one lever contact. It is evident that the subjects of the response-terminated omission group contacted the lever at substantially higher levels than did the fixedtrial omission subjects throughout the 35 sessions $(p<.05){ }^{2}$ This difference emerged as early as Session 2 .

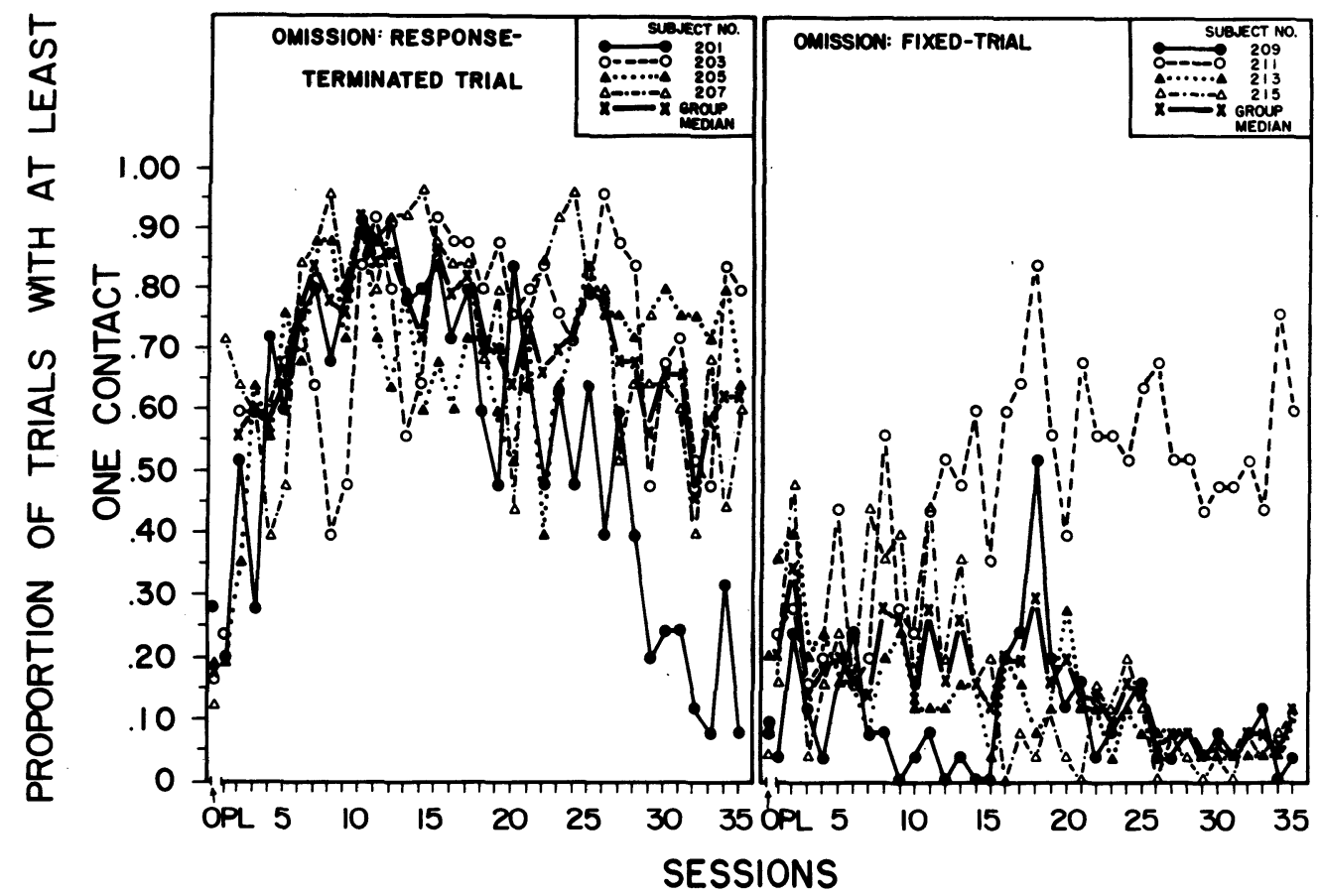

Figure 1. Proportion of trials with at least one lever contact for each omission subject. The median of each group is also shown. Data points to the left of Session 1, labeled "OPL," indicate the mean of the last five operant-level sessions. 


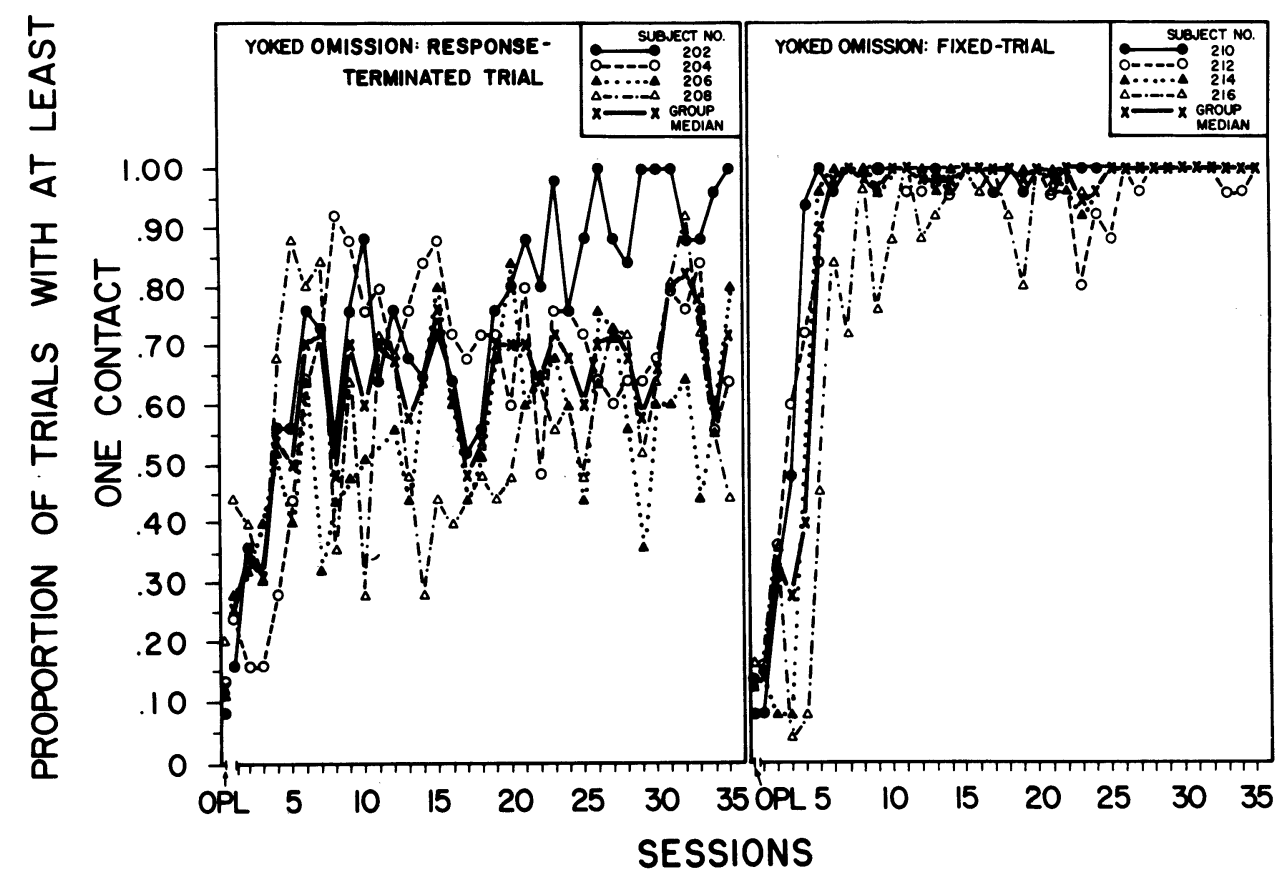

Figure 2. Proportion of trials with at least one lever contact for each yoked control subject. The median of each group is also shown. Data points to the left of Session 1, labeled "OPL," indicate the mean of the last five operant-level sessions.

Moreover, there was virtually no overlap between the groups during the first 20 sessions. Thereafter, some decrements can be seen for subjects in both groups. However, over the last five sessions mean response probability for the response-terminated group was .59 , as compared to .18 for fixed-trial subjects.

Figure 2 shows the response probability for each of the yoked partners of the two omission groups. The probability of responding for yoked controls of the fixed-trial omission subjects was uniformly high and exceeded that of their omission partners throughout training $(p<.05)$. Three of the four yoked subjects reached the 1.0 probability level by Session 6, whereas the fourth subject (216) reached this level on Session 11.

A markedly different pattern was noted for the yoked partners of response-terminated omission subjects. The responding of these subjects vacillated but gradually increased toward the end of training. During the first half of training (Sessions 1-18), the response probability of omission subjects surpassed that of yoked subjects $(p<.05)$, but no differences were observed between these groups during the last half of training (Sessions 19 $35)^{3}$

No differences were observed between the two random control procedures $(\mathrm{p}>.05)$. Most subjects in both procedures responded on less than .30 of the trials although there were several subjects with high response levels.

\section{DISCUSSION}

Autoshaping has prompted numerous reevaluations of twofactor learning theory. Most importantly, there has been an in- creased emphasis on the role of Pavlovian relations in the acquisition and maintenance of response systems previously thought to be under the primary control of instrumental contingencies (e.g., Hearst \& Jenkins, 1974; cf. Jenkins, 1977).

This assessment of autoshaping depends importantly upon the data generated using the omission procedure. The present data indicate, however, that at least with respect to this preparation, trial offset functions as a conditioned reinforcer and contributes substantially to responding. One may observe, for example, that in the fixed-trial omission procedure, where responses were adventitiously paired with trial offset, levels of responding were comparable only to those engendered by the random control procedures. These data serve as a reminder that it may be premature to regard autoshaping as essentially incompatible with interpretations that emphasize responsereinforcer relations.

In addition to these omission training data, the comparison between omission subjects and their yoked controls is of interest. It should be remembered that, as these two groups were equated in terms of S-S* pairings, any differences between them must be accounted for by the possible adventitious pairings between responses and food for yoked subjects. In the case of fixedtrial subjects, the probability of responding for yoked controls exceeded that of omission subjects throughout training.

However, a reversal of this pattern was observed in the case of the response-terminated subjects where the probability of responding for omission subjects exceeded that of their yoked partners early in training and was comparable to that of yoked subjects during the latter stages of training. In assessing these data one should bear in mind that yoked subjects would have to respond during a trial before the first response emitted by their omission partner. As a result, yoked-omission/responseterminated subjects experienced varying opportunities to respond during training, a factor which may have been responsible for their relatively low levels of responding as compared to those observed for yoked-omission/fixed-trial subjects.

Quite apart from the appropriate characterization of these findings, it is important to note that in two respects they stand in contrast to those reported with pigeons. First, as observed previously, studies seeking to define the role of trial 
offset in omission training have yielded conflicting results (cf. Brownstein \& Balsam, 1975; Herrnstein \& Loveland, 1972; Hursh et al., 1974; Peterson et al., 1977; Schwartz, 1972). Yet the present data provide a systematic demonstration of trial offset effects. A second difference concerns the finding that fixed-trial omission procedures apparently support relatively substantial levels of responding in pigeons but failed to do so in the present case (e.g., Schwartz \& Williams, 1972).

It is reasonable to suggest that the discrepancies between the present data and prior work are due to the preparations used (i.e., rats and retractable levers as compared to pigeons and visual stimuli). Moreover, it has been suggested that mammals and avian species may differ in their sensitivity to R-S* and S-S* contingencies (e.g., Gray, 1975). Yet two observations argue against characterizing these differences in that manner. For one, reports of successful response maintenance using fixed-trial omission procedures with pigeons have not been unequivocally confirmed (e.g., Griffin \& Rashotte, 1973; Hursh et al., 1974; Powell \& Kelly, 1976). Also, despite the rather substantial interest shown in autoshaping using pigeons, relatively few studies have investigated the role of trial offset in omission training or the more comprehensive issue of the extent to which omission-trained responses exhibit sensitivity to their consequences.

\section{REFERENCES}

Brownstein, A. J., \& Balsam, P. D. A search for conditioned reinforcement effects in negative automaintenance of key pecking. Bulletin of the Psychonomic Society, 1975, 6. 165-168.

Fleshler, M., \& Hoffman, H. S. A progression for generating variable interval schedules. Journal of the Experimental Analysis of Behavior, 1962, 5, 529-530.

GAmzU, E., \& Schw AM, E. Autoshaping and automaintenance of the key-press response in squirrel monkeys. Journal of the Experimental Analysis of Behavior, 1974, 21, 361-372.

GRAY, J. A. Elements of a two-process theory of learning. London: Academic Press, 1975.

Griffin, R. W., \& Rashotte, M. E. A note on the negative automaintenance procedure. Bulletin of the Psychonomic Society, 1973, 2, 402-404.

Hearst, E., \& Jenkins, H. M. Sign tracking: The stimulusreinforcer relation and direction action. Austin, Tex: Psychonomic Society, 1974.

Herrnstein, R. J., \& Loveland, D. Food avoidance in hungry pigeons and other perplexities. Journal of the Experimental Analysis of Behavior, 1972, 18, 369-383.

Hursh, S. R., Navarick, D. J., \& Fantino, E. "Automaintenance": The role of reinforcement. Journal of the Experimental Analysis of Behavior, 1974, 21, 117-124.

Jenkins, H. M. Sensitivity of different response systems to stimulus-reinforcer and response-reinforcer relations. In $\mathrm{H}$.
David \& H. M. B. Hurwitz (Eds.), Operant-Pavlovian interactions. Hillsdale, N.J: Lawrence Erlbaum, 1977.

Locurto, C., Terrace, H. S., \& Gibbon, J. Autoshaping, random control, and omission training in the rat. Journal of the Experimental Analysis of Behavior, 1976, 26, 451-462.

Peterson, M. R., Lyon, D. O., Stone, W., \& Scott, W. The role of conditioned reinforcement in the acquisition and maintenance of omission responding. Psychological Record, 1977, 27, 235-254.

Powell, R. W., \& Kelly, W. Responding under positive and negative response contingencies in pigeons and crows. Journal of the Experimental Analysis of Behavior, 1976, 25, 219-225.

Rescorla, R. A. Pavlovian conditioning and its proper control procedures. Psychological Review, 1967, 74, 71-80.

SCHWARTZ, B. The role of positive conditioned reinforcement in the maintenance of key pecking which prevents the delivery of primary reinforcement. Psychonomic Science, 1972, 28, 277-278.

Schwartz, B., \& Williams, D. R. The role of the responsereinforcer contingency in negative automaintenance. Journal of the Experimental Analysis of Behavior, 1972, 17, 351-357.

SIEGEL, S. Nonparametric statistics for the behavioral sciences. New York: McGraw-Hill, 1956.

Stiers, M., \& Silberberg, A. Lever-contact responses in rats: Automaintenance with and without a negative response-reinforcer dependency. Journal of the Experimental Analysis of Behavior, 1974, 22, 497-506.

Williams, D. R., \& Williams, H. Automaintenance in the pigeon: Sustained pecking despite contingent non-reinforcement. Journal of the Experimental Analysis of Behavior, $1969,12,511-520$.

\section{NOTES}

1. Responses were not recorded during the first $.50 \mathrm{sec}$ of each trial. This procedure was followed to eliminate the possibility that contact with the lever might occur during insertion of the lever into the chamber.

2. Statistical tests were conducted using the randomization test (Siegel, 1956).

3. Results comparable to these in every respect were obtained from subjects that had experienced 25 sessions of fixed-trial $(15-\mathrm{sec})$ autoshaping prior to placement on the omission or yoked procedures.

(Received for publication March 1, 1978.) 